\title{
La communauté Sourde et les soins de santé
}

\author{
Mary Malebranche MD, Kevin Morisod MD, Patrick Bodenmann MD
}

Citation : CMAJ 2020 December 14;192:E1809. doi : 10.1503/cmaj.200772-f

Voir la version anglaise de l'article ici : www.cmaj.ca/lookup/doi/10.1503/cmaj.200772

\section{1} Faire partie de la communauté Sourde n'est pas synonyme d'avoir une déficience auditive

Environ 357000 Canadiens et Canadiennes se disent membres de la communauté Sourde (avec la majuscule), une minorité culturelle et linguistique distincte qui utilise la langue des signes pour communiquer et pour qui la surdité est un état, et non un handicap à corriger ${ }^{1}$. En revanche, autour de 3,2 millions de Canadiens et Canadiennes ont une déficience auditive, parfois grave ou profonde (on les dit « sourds » avec la minuscule).

2

Les membres de la communauté Sourde ont de moins bons résultats de santé mentale, cardiovasculaire et sexuelle que les personnes non Sourdes ${ }^{2,3}$

Étant donné leur accès limité aux médias de masse conçus pour les entendants, aux conversations environnantes et aux messages de santé publique, les membres de la communauté Sourde sont 6,9 fois plus susceptibles que les personnes non sourdes d'avoir une littératie en santé inadéquate $^{4}$. Les Sourds utilisant la langue des signes ont beaucoup moins de connaissances en santé cardiovasculaire que ces dernières ${ }^{4}$.

\section{3}

Ce ne sont pas toutes les personnes atteintes de perte auditive grave à profonde qui parlent couramment la langue des signes Il faut s'enquérir auprès de chaque personne du mode de communication qu'elle privilégie, par exemple la lecture labiale, la communication écrite, les aides visuelles ou la langue des signes. Si une personne préfère la langue des signes, un interprète devrait être mis à sa disposition ${ }^{4}$. Il vaut mieux éviter de présumer que les personnes Sourdes lisent bien sur les lèvres, puisqu'il arrive qu'elles ne comprennent que $30 \%$ des conversations.

\section{Les avancées technologiques peuvent servir à améliorer la communication avec les personnes Sourdes}

L'intégration aux soins cliniques des innovations en interprétation de la langue des signes, comme KinTrans (www.kintrans.com) et Sign-Speak (sign-speak.com), a un potentiel considérable pour améliorer la communication avec les usagers de la langue des signes ${ }^{5}$.

La disponibilité des implants cochléaires a soulevé des préoccupations à propos de leurs répercussions sur l'identité culturelle Sourde

Bien que les implants cochléaires soient associés à une amélioration de l'audition et du développement de la langue parlée, leur effet sur l'identité culturelle, la participation sociale et le bien-être des personnes Sourdes demeure sujet de controverse. Par exemple, l'intégration des enfants Sourds ayant des implants cochléaires dans des écoles régulières a causé un déclin des écoles spécialisées pour les sourds ${ }^{6}$.

\section{Références}

1. Statistics on Deaf Canadians. Ottawa: The Canadian Association of the Deaf - Association des Sourds du Canada; 2015. Accessible ici : cad.ca/issues-positions/statistics-on-deaf-canadians/ (consulté le 15 avril 2020).

2. Fellinger J, Holzinger D, Pollard R. Mental health of deaf people. Lancet 2012;379:1037-44.

3. Emond $\mathrm{A}$, Ridd $\mathrm{M}$, Sutherland $\mathrm{H}$, et al. Access to primary care affects the health of Deaf people. Br J Gen Pract 2015;65:95-6.

4. McKee MM, Paasche-Orlow MK, Winters PC, et al. Assessing health literacy in deaf American sign language users. $J$ Health Commun 2015;20:92-100.

5. Smeijers A, Ens-Dokkum MH, van den Bogaerde B, et al. Availability of specialised healthcare facilities for deaf and hard of hearing individuals. International Journal on Mental Health and Deafness 2018;4:14-27.

6. Chapman M, Dammeyer J. The relationship between cochlear implants and deaf identity. Am Ann Deaf 2017;162:319-32.

Intérêts concurrents : Aucun déclaré.

Cet article a été révisé par des pairs.

Affiliations : Département des vulnérabilités et médecine sociale (Malebranche, Morisod, Bodenmann), Centre universitaire de médecine générale et santé publique, Université de Lausanne, Lausanne, Suisse; Département de médecine (Malebranche), Faculté de médecine Cumming, Université de Calgary, Calgary, Alb.

Propriété intellectuelle du contenu : Il s'agit d'un article en libre accès distribué conformément aux modalités de la licence Creative Commons Attribution (CC BY-NCND 4.0), qui permet l'utilisation, la diffusion et la reproduction dans tout médium à la condition que la publication originale soit adéquatement citée, que l'utilisation se fasse à des fins non commerciales (c.-à-d., recherche ou éducation) et qu'aucune modification ni adaptation n'y soit apportée. Voir : https://creativecommons.org/ licenses/by-nc-nd/4.0/deed.fr.

Remerciements : Les auteurs sont reconnaissants pour la contribution intellectuelle du $D^{r}$ Laurent Frikart, un otorhinolaryngologiste qui travaille auprès de personnes Sourdes ou ayant une déficience auditive à Lausanne, en Suisse. Ils tiennent aussi à souligner celle de Stéphane Beyeler, un usager Sourd de la langue des signes qui s'identifie comme membre de la communauté Sourde de Lausanne et est directeur régional francophone de la Fédération Suisse des Sourds. Tous deux ont examiné le contenu d'une version révisée du manuscrit et fourni du contenu intellectuel précieux.

Correspondance : Mary Malebranche, mary.malebranche@ucalgary.ca 